\title{
COLLABORATION BAROMETER - DEVELOPMENT OF A TOOL FOR MEASURING COLLABORATION DURING DESIGN AND CONSTRUCTION
}

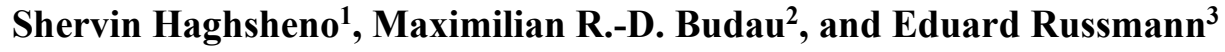

\begin{abstract}
Many concepts in Lean Construction are based on collaboration. In project delivery models such as Integrated Project Delivery (IPD), which relies on a high degree of integration, the success of the project depends particularly on the degree of collaboration between the project participants. But even in traditional project delivery models such as design-bid-build, construction management at risk or design-build the degree of collaboration can have a strong influence on project success.

While many project parameters such as costs, deadlines, quality, changes or risks are measured and controlled within the framework of project management of construction projects, hardly any focus is placed on measuring and controlling the important factor of collaboration between the project participants in a project.

Starting with the basics on collaboration, this paper describes the development of a tool called "Collaboration Barometer", which can be used to measure the degree of collaboration between the participants in a construction project and shows how the results are processed and what benefits are derived from them. The paper also includes experiences with the first applications of the tool and gives recommendations for its use.
\end{abstract}

\section{KEYWORDS}

collaboration, culture, project controlling, project delivery

\section{INTRODUCTION}

In the past, the willingness to cooperate was an essential basis for the construction of buildings. Over the last 20 years, the willingness to cooperate in the construction industry seems to have steadily decreased (Merkle 2017). This is partly due to the forms of project delivery that are predominantly used, which aim in particular to optimize costs and thus increasingly lead to conflicts during execution (Girmscheid 2010). Conflicts in turn reduce the willingness to cooperate even more. The exact extent of the influence is unknown here. This is again due to the fact that the degree of cooperation, in contrast to costs, deadlines and qualities, cannot be measured directly.

1 Prof., Director, Institute of Technology and Management in Construction, Karlsruhe Institute of Technology, Germany, shervin.haghsheno@kit.edu, orcid.org/0000-0002-0602-6370

2 Research Associate, Institute of Technology and Management in Construction, Karlsruhe Institute of Technology, Germany, maximilian.budau@kit.edu, orcid.org/0000-0002-2572-1176

3 Research Associate, Institute of Technology and Management in Construction, Karlsruhe Institute of Technology, Germany, orcid.org/0000-0002-5919-3279 
Within the framework of a research project at the "Karlsruhe Institute of Technology" (KIT), a method was developed that allows to record the degree of collaboration in a project and to present the results to the participants in a clear form. In this paper, the method as well as experiences from the first application are presented. This research is particularly important in the context of Lean Construction, since many aspects of lean construction are based on collaboration (Schöttle et al. 2014).

In order to present the topic of collaboration from different perspectives, the first step will be to present the results of a literature research on the topic of "collaboration". According to the above definition, the organizational as well as cultural aspects will be addressed. In the next step, already existing approaches for the measurement of collaboration in construction projects are presented. Subsequently, the development of the collaboration measurement instrument is explained and the experiences with the first application are presented.

\section{COLLABORATION}

\section{Collaboration vs. CoOperation}

In literature, the terms collaboration and cooperation are often used synonymously. Collaboration goes beyond cooperation. Collaboration describes the common vision to create a common project organisation with a jointly defined structure and to create a project culture based on trust and transparency. Since the value for the customer is the central focus here, the focus in the following will be on collaboration. (Schöttle et al. 2014) Collaboration is described from different perspectives and especially experiences from outside the construction industry are taken into account in order to guarantee a broad overview on the topic of collaboration.

\section{Collaboration From a Socio-Cultural Perspective}

Every person has the incentive to behave selfishly and to be concerned about his own well-being. At the same time collaboration is the basis of human existence. (Axelrod 2000) Fundamental to the development of collaborative behaviour is the building of interpersonal bonds. Such bonds are strengthened by repeated interactions of the participants. (Axelrod 2000) Likewise, repeated interaction alone is not sufficient to establish collaboration between the participants. (Axelrod 2000)

There is a connection between collaboration and trust. The expectation of collaborative behavior is called trust. When others exploit a collaborative attitude of a participant, their position is weakened and abused. (Bierhoff 1998)

In reality, an interaction between people is usually not unique. An individual's strategy of consistently behaving uncooperative will have negative consequences. In this case, the other persons involved have no interest in showing collaborative behaviour, so that no collaboration occurs and losses are to be expected on all sides. The 'tit-for-tat' strategy describes the behaviour that proves to be most advantageous. The strategy says to behave collaboratively at the first decision. In the next decision, the last reaction of the other participants is reflected and copied. An uncollaborative behaviour is reacted to with the same behaviour. Similarly, in the case of collaborative behavior, a collaborative reaction is carried out. (Bierhoff 1998)

In empathic collaboration, the participants try to put themselves in the other person's shoes. In addition to collaboration, a common goal is also sought. The possibility is opened up to work together to achieve the goals. (Spieß 1998a) In contrast to empathic 
collaboration, in pseudo-empathic collaboration compassion and an understanding of the situation are feigned. (Tries and Reinhardt 2008)

The natural collaboration can be observed daily when dealing with friends and acquaintances. It is characterized by a strongly cooperative attitude. This form of collaboration is associated with emotions that exist or are intuitively created in relation to others. (Tries and Reinhardt 2008)

\section{Collaboration From a Organizational Perspective}

Collaboration between companies or departments of a company can be essential for mutual success. There are conditions that promote development in a positive direction. On the one hand, there must be equality between the actors and on the other hand, both the willingness and the expectation to collaborate jointly must prevail. (Bierhoff 1998)

Since trust and collaboration are intertwined, it is beneficial to create an atmosphere of trust within and between companies. Honest and loyal behaviour and the opportunity to discuss problems are the basis for a trusting relationship. (Bierhoff 1998)

Between companies, departments or even individual persons, the respective work is linked. It is more advantageous and more efficient to jointly pursue higher-level goals. (Bierhoff 1998)

Within companies, a cooperative attitude is taken for granted. (Nerdinger 1998)

Collaboration is an important topic in the economy. It can be seen not only in the behaviour towards work colleagues, but also in inter-company relations. A possible profit or loss plays a significant role in this. When deciding to collaborate, it is not possible to predict how the collaborating partner will react. If the partner also intends to collaborate, both will gain from the situation. If the partner refuses to collaborate in order to pursue his own profit strategies, there is a possibility that strong losses will be incurred by the other parties. In this case, an uncollaborative behaviour is the better choice for the parties involved, as there is no possibility of abusing a collaborative attitude. (Bierhoff 1998)

Conflicts can be resolved through communication. Communication forms the basis for effective collaboration. To make collaboration successful, defined goals and a time lead are required. The participants must get to know each other and build trust. (Spieß 1998b)

In a coordinated approach, each actor has its own task or goal. The pattern of action is therefore predetermined and agreed between the actors. There is no need for interaction between the participants. The prerequisite for this is a collaborative working method. The participants work towards a common goal. (Langemeyer 2015)

Construction companies are mainly organised on a project-oriented basis. The majority of a construction company consists of project groups. There are important internal company factors that are fundamental to project management. These include the motivation of employees, their sense of community, the exchange of information and the authority to make decisions. Other factors at the project management level are clear common goals, standardization of processes and the competencies of the project management. (Spang 2006) Collaboration at project level supports the integration and thus helps to achieve the project goals. (Fischer et al. 2017)

Schöttle et al. (2014) summarize the socio-cultural and organizational as well as other aspects of collaboration in distinction to cooperation and autonomous or coordinated work in the following figure. 


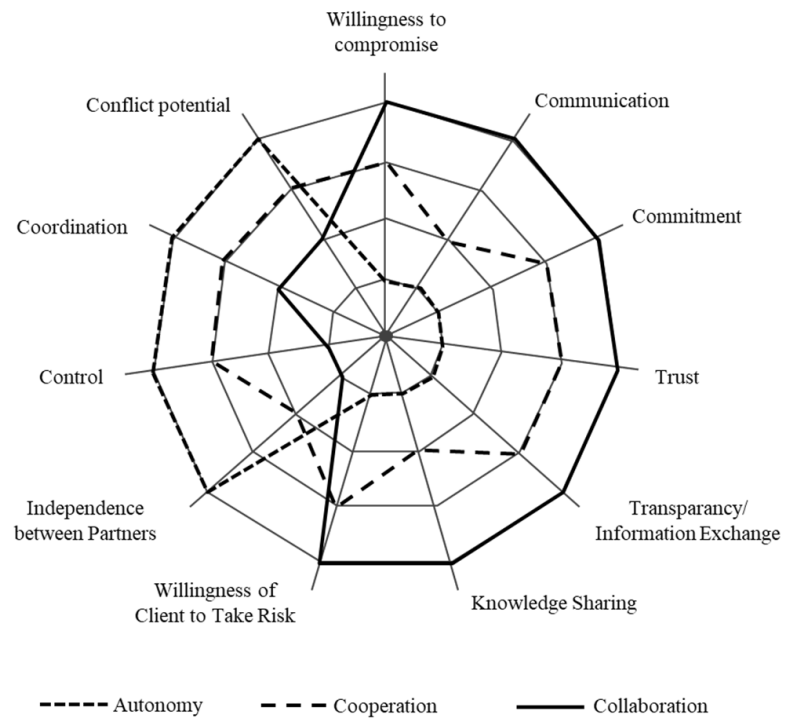

Figure 1: Compromise the terms cooperation and collaboration (Schöttle et al. 2014)

\section{MEASUREMENT OF COLLABORATION - STATE OF RESEARCH AND PRACTICE}

Research and practice have recognized the importance of collaboration for successful project management. Anvuur (2008), for example, dealt in great detail with the influence of collaboration and strategies for optimizing it as well as individual methods for measuring it. In particular, the method according to Phua (2004) should be mentioned here, which attempts to make statements about the degree of collaboration by means of a simple survey.

Abdirad and Pishdad-Bozorgi (2014) identified success criteria for a successful collaboration in IPD projects as well as potential evaluation criteria on the basis of an extensive literature research. Within the scope of this literature research, they also considered in particular literature without direct reference to the construction industry.

Beyond that, Walker and Lloyd-Walker (2019) shows how a concrete collaboration barometer as a visualization tool for benchmarking can look like.

Bales uses a similar method in his "Interaction Process Analysis" (IPA). Here, however, he relies on a neutral third party, who describes the interactions between the participants with the help of observations in order to create a basis for control on this basis. (Emmitt and Gorse 2007)

In April 2018, the „Österreichische Bautechnik Vereinigung” (öbv) published the leaflet "Cooperative project management". This contains a short description of the analysis and control tool "KOOPQuickCheck". The tool offers a possibility to display the current collaboration in a construction project and to show the development of the collaboration over the duration of the project.

(Österreichische Bautechnik Vereinigung 2018)

The measurement of the collaboration is realized by a questionnaire. The presentation of the results of a survey is done by a scatter diagram and several collaboration indices. (Österreichische Bautechnik Vereinigung 2018) 
In addition, there are other studies that deal with the performance measurement of partial aspects of cooperation or consider cooperation as part of a comprehensive performance measurement. (e.g. Pocock et al. 1996, El Asmar 2012)

\section{DEVELOPMENT OF THE COLLABORATION BAROMETER}

\section{METHOD}

At the beginning of the development of an instrument to describe the level of collaboration and assess it, the requirements for this measuring instrument must be defined.

In the next step the measuring instrument can be designed. This is done in the form of a control loop. For this purpose, the measuring method is defined at the beginning. Subsequently, the measurement contents are determined and validated in pilot surveys. The results of the validation are in turn incorporated along the control loop.

In the final step, the finalized measurement instrument can be used in construction projects. These three steps of the method are shown in the following figure 2. This approach is based on proven development methods such as the "Worldwide Solution Design and Delivery Method" (WSDDM) (e.g. Noack and Schienmann 1999) used by the software industry. It should be noted that this control cycle must be repeated for each new implementation of the collaboration barometer. This is the only way to ensure that the Collaboration Barometer fits to the project.

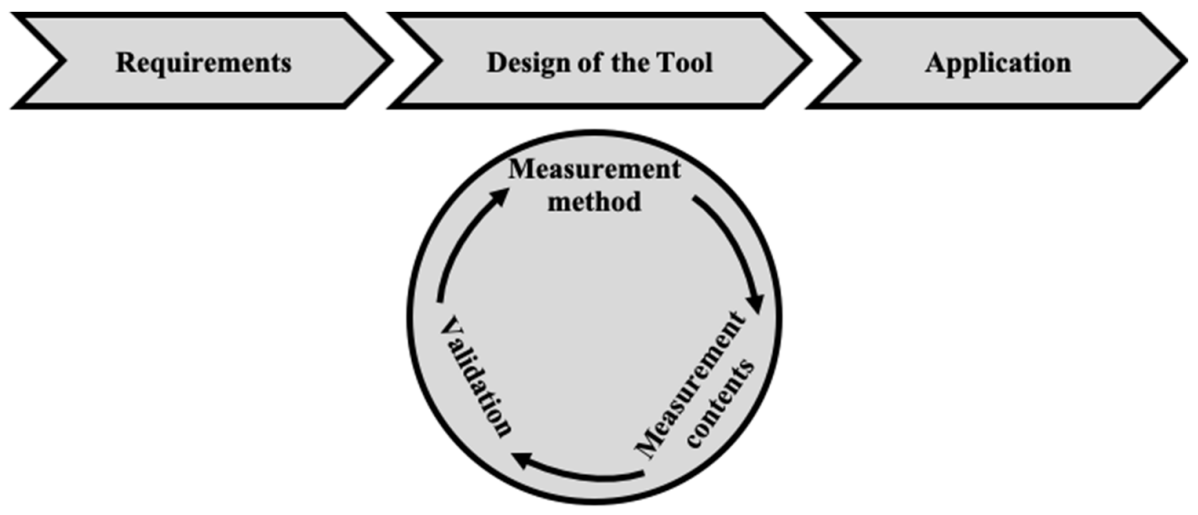

Figure 2: Method for creating the measuring instrument

In the following subchapters, the first two steps of the methodology are discussed. The experience gained from the implementation of the tool during the first projects serves as a basis for this. The concrete experiences within the third step are presented in the following chapter. As the projects are not yet completed, no further information on these projects may be given for data protection reasons.

\section{REQUIREMENTS}

At the beginning the requirements for the model were discussed with the project partners. For the previous applications the requirements could be summarized as follows. The aim of the measurement instrument is to provide a data basis on which statements on collaboration can be made. In addition, the barometer must help to identify aspects of the collaboration that show potential for improvement. 
The measuring instrument should be as easy to use as possible and should not create disproportionate additional work for the project participants. In addition, the results should be clearly presented to allow a quick assessment of the situation.

\section{DESIGN OF THE MEASURING InSTRUMENT WITH REgaRd TO THE Measuring Method}

Every form of control requires a data basis. The previous explanations show, among other things, that the effect of collaboration can only be quantified to a limited extent. It is therefore not possible to draw conclusions about collaboration from a target figure, such as the project results. (Abdirad and Pishdad-Bozorgi 2014)

As will be shown in the following, the measurement instrument is used to obtain qualitative statements on aspects of collaboration in particular, in order to make a statement on the degree of collaboration in a project on this basis. In the following, the most suitable method of data acquisition is derived.

One of the first experiences from discussions with people involved in the pilot projects was that within a construction project it is difficult to make appointments with a sufficient number of participants to conduct an oral survey. For the interviewer, the time required is considerable. In addition, the interviewer can influence the process. Due to these disadvantages, an oral interview is not suitable for the collaboration measurement instrument.

In a written survey, questionnaires are sent out, filled in by the participants and returned. A manual input of data for the evaluation is prone to errors. With a large number of project participants, data entry takes a lot of time.

Therefore, the method of an online survey was chosen for data collection. There is a temporal and spatial independence. This is advantageous because the participants have the possibility to carry out the survey in their company, at home or on the construction site. The answers of the respondents are made available independently of influence and at the same time. There is no need for manual input as the data is available in digital form. This also shortens the time needed to evaluate the results. Due to these advantages, a form of online survey will probably also be used as standard for every further application.

\section{DESIGN OF THE MEASUREMENT INSTRUMENT WITH REgaRD TO THE CONTENT}

Based on the results of the literature study on collaboration in general (see above) and the state of the research on the measurement of collaboration in civil engineering, under consideration of the project-specific conditions individual factors influencing collaboration were derived and classified. Figure 3 shows this as an example for one of the first collaboration barometer pilot projects. In other project applications, only slight adjustments have been made so far. The basic structure has been retained. Thus the structure shown is generally valid. The starting point was in particular the collaboration factors identified by Schöttle et al (2014) and Abdirad and Pishdad-Bozorgi (2014). In the following, some important aspects are presented first, which serve as a basis for the development of influencing factors.

The personal perception of the respective project participants plays a major role in their performance and their behaviour towards their fellow human beings in their environment.This is reflected in the success of the construction project, if these perceptions are positive. It is essential both that one's own performance is satisfactory and 
that it is appreciated by those involved. Mutual respect for the work done is part of good cooperation and creates the basis for trust and good communication.

Communication is an essential basis of collaboration. Successful communication between project participants is essential. A common solution finding is favoured by it.

The consideration of different opinions shows the will to work out solutions that are satisfactory for all. Empathic collaboration is promoted by developing an understanding the respective situations of those involved in the project and by including them in one's own viewpoint. Communicative behaviour is improved by comparing the different objectives and jointly agreeing on the decisions to be taken.

Within a construction project there are a multitude of different participants. Clearly defined responsibilities help to avoid conflicts due to decisions not made in time.

There are numerous interfaces between the parties involved. A successful cooperation is therefore characterized by intensive coordination.

In order to make the right decisions, relevant data is required to varying degrees. Two factors are important. On the one hand, it is relevant that important information is openly communicated and on the other hand that required information is communicated in time. If information is not passed on or is passed on late, there is a risk that trust and the will to collaborate between the parties involved will decline.

It is necessary that those involved react flexibly to changes and often make short-term decisions. Deadline commitments must be reliably met. Errors and delays in the construction process are thus avoided. The potential for conflict decreases.

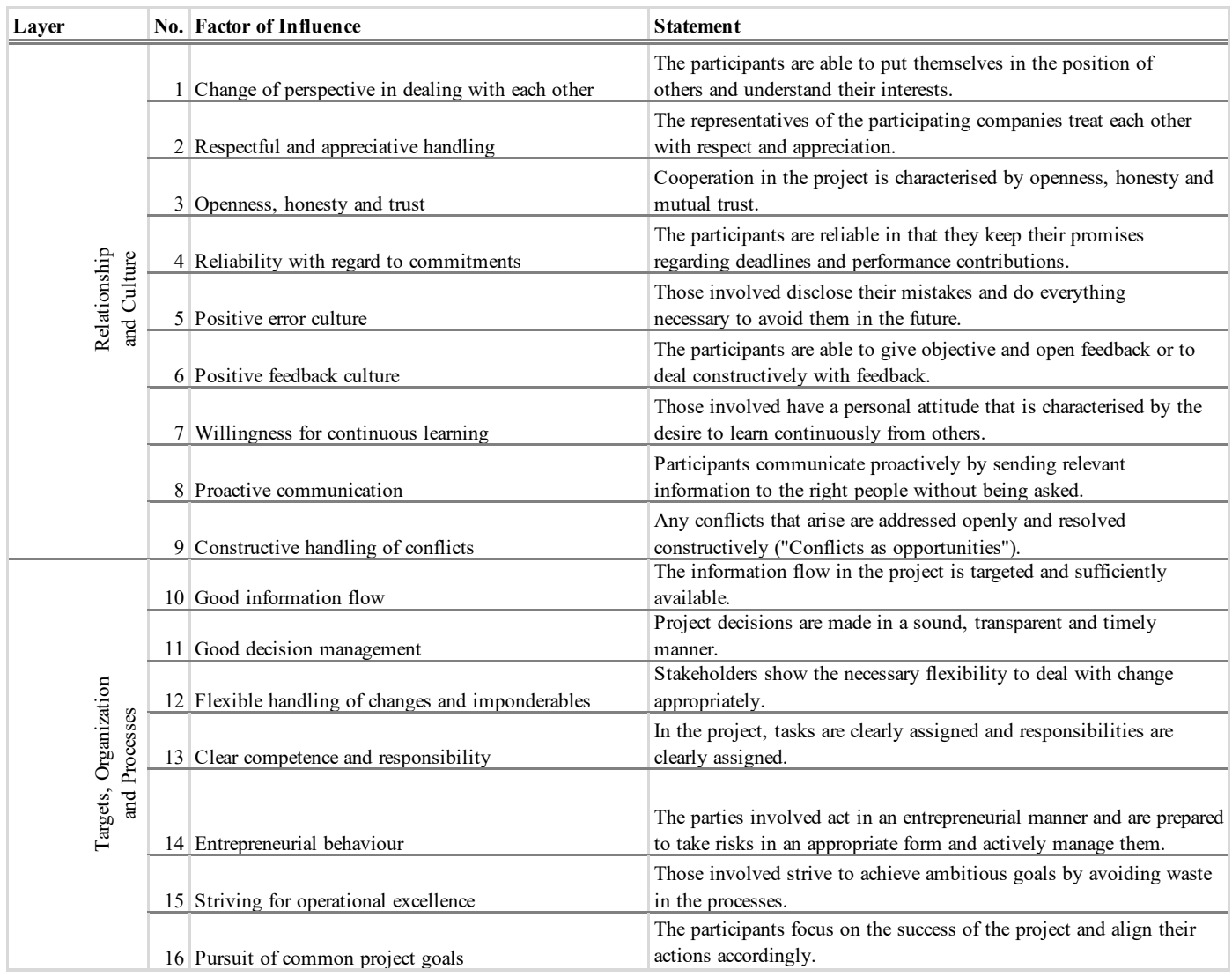

Figure 3: Illustration of the influencing factors in the collaboration barometer 
Looking at collaboration from a socio-cultural and organizational perspective shows that trust plays a decisive role in collaboration. A cooperative attitude of the parties involved is expected. Honesty and the opportunity to discuss problems openly promote a trusting relationship between the project participants.

A cross-company sense of community within the project shows that common goals are being pursued. This creates a stronger feeling of identification of the participants with the project. The equal treatment of actors also steers the cooperation in a positive direction.

Conflicts can be an obstacle to effective cooperation if they are not dealt with early on. In turn, statements about the basic collaborative attitude can be derived from the behaviour during conflicts.

It is important that the project participants behave collaboratively even during the course of a conflict. Constructive and result-oriented work is done to develop a joint solution. This is not possible if different views on the matter are applied to the relationship between the project participants.

Influencing factors can be derived from the aspects of collaboration presented. Figure 3 shows the influencing factors, including a description, that arose at the end of a validation process in the course of the aforementioned pilot project.

The validation of the collaboration barometer took place in the form of pilot surveys, in which the survey design was tested and adapted. The influencing factors were divided into two areas as shown in figure 3. The first area comprises the influencing factors that address in particular the "relationship and culture". The second area comprises the influencing factors that can be summarised under the aspects "targets, organization, processes". The measuring instrument thus provides for a division into aspects which, on the one hand, cover the cultural level and, on the other, are related to the system of project management and thus cover the organizational level.

\section{DESIGN OF THE MEASURING INSTRUMENT WITH REGARD TO THE IMPLEMENTATION}

Different areas of collaboration will be covered in the survey. Not all areas are equally relevant for the participants. For example, good information flow may play a greater role in successful collaboration than other factors influencing collaboration. Besides the project-specific design of the collaboration barometer a project-specific evaluation of the relevance of the influencing factors (figure 3 ) is necessary to assess collaboration in a construction project.

Thus, a two-part, standardized questionnaire is developed, which records the relevance of the influencing factors in general in the first part and their project-specific characteristics in the second part. The relevance of the factors is determined by the participants. The weighting should be independent from the project. To avoid mutual influence, participants are expected to answer both parts of the questionnaire independently of each other. A separate consideration of relevance and the characteristics of the factors is reinforced by the use of different response scales.

The answers are given independently. The questionnaire is designed to be comprehensible and simple. Four scale levels are used to assess the project-specific characteristics of the influencing factors. A unipolar scale is used. The gradation is made by verbal designations. The differentiations develop from a negative attitude "Does not apply at all" to an approving attitude "Fully applies". In the gradations in between, attention is paid to an approximate equidistance. There is no neutral centre. A tendency 
in one of the two directions is thus achieved, so that the collaboration is evaluated by the participants as negative or positive. The consideration of a lack of opinion is realized with the help of the additional category "No statement".

A five-level response scale is used to indicate relevance. The middle level gives the possibility to consider a statement as neither important nor unimportant. To strengthen the connection to the project-specific expression, a unipolar scale with verbal gradations is used. The designations develop from "no relevance" to "highest relevance". It is assumed that all participants in a construction project have an opinion on the different areas of collaboration. There is a need for respondents to engage with the issues. A category that expresses a lack of opinion makes it possible to reduce the amount of work involved. This category is not used in the questionnaire.

An influence by the first-mentioned answer category is possible with rating scales. When answering the items in a project-specific manner, the system starts with "Does not apply at all". The failure of the collaboration is more likely to be recognized.

The questionnaire contains critical elements such as behaviour in conflicts. It is possible that a personalised survey would influence the participants. This could possibly lead to a distortion of the results. The survey is therefore anonymous.

The survey will be extended by an item that allows the naming of suggestions for improvement for a good cooperation in the construction project. An open answer format allows participants to express themselves freely without being bound to an answer scale.

Two further questions clarify the affiliations of the different participants to possible project groups. This increases the possibility of evaluation differentiated by project groups.

Conducting the survey is only one step in the overall process of applying the "Collaboration Barometer". The whole process is shown in Figure 4.

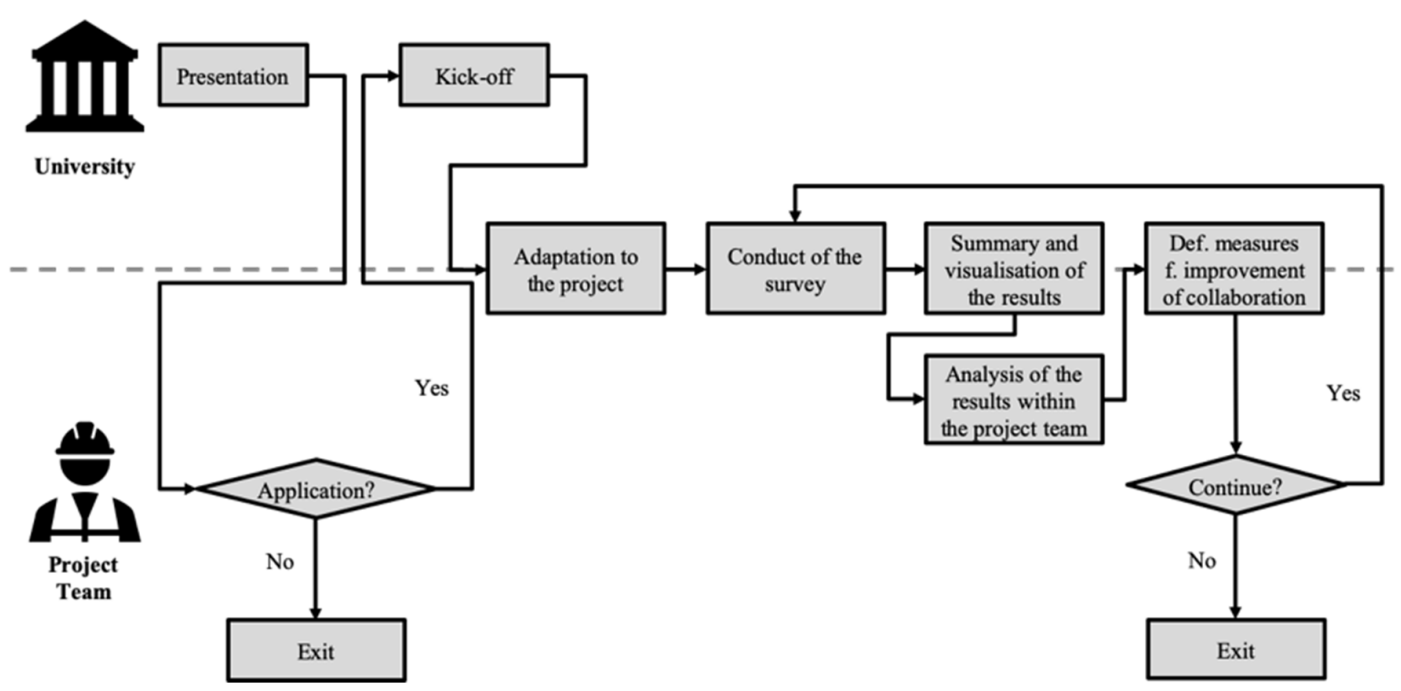

Figure 4: Process for adopting the measuring instrument

At the beginning of the process, a presentation of the Collaboration Barometer is given to the project leaders. Afterwards, they can decide for or against the application in a project or across companies. If a decision for the application is made, the team concerned must be informed in advance about the measurement instrument in order to create an understanding and to achieve the highest possible number of participants in the implementation. In this course, the barometer can still be adapted to the project. For 
example, it is possible to form special project groups in order to structure the evaluation according to these groups. After the subsequent execution of the survey, the collected data must be processed again. This includes determining corresponding average values for all participants and calculating them to form an overall value. The results for the individual influencing factors as well as the aggregation to an overall value for the project and the presentation of the project progress are visualized and compiled in a report with an executive summary. The results of a survey must then be discussed within the project team, ideally with the support of a moderator, in order to agree on steering measures if necessary. The survey with the subsequent evaluation is repeated at a regular rhythm until the end of the project, which also allows the development of the degree of cooperation during the course of the project to be recorded.

\section{EXPERIENCE WITH THE APPLICATION OF THE COLLABORATION BAROMETER}

The measuring instrument in the present version was developed in 2019 by the "Institute of Technology and Management in Construction" (TMB) at the KIT and has already been applied in several projects under the name "Collaboration Barometer".

In particular, it turned out that the measuring instrument can be applied in all project delivery models. Figure 5 shows an exemplary extract from the evaluation of a collaboration barometer. In the upper half of the excerpt the collaboration index is shown in different ways. The Collaboration Index results from the calculation of the projectspecific manifestations of the influencing factors weighted with the respective relevance. On the left-hand side, the index is divided into categories, and on the right-hand side, it is shown over time. The lower part of the graphic shows the individual values for each influencing factor.

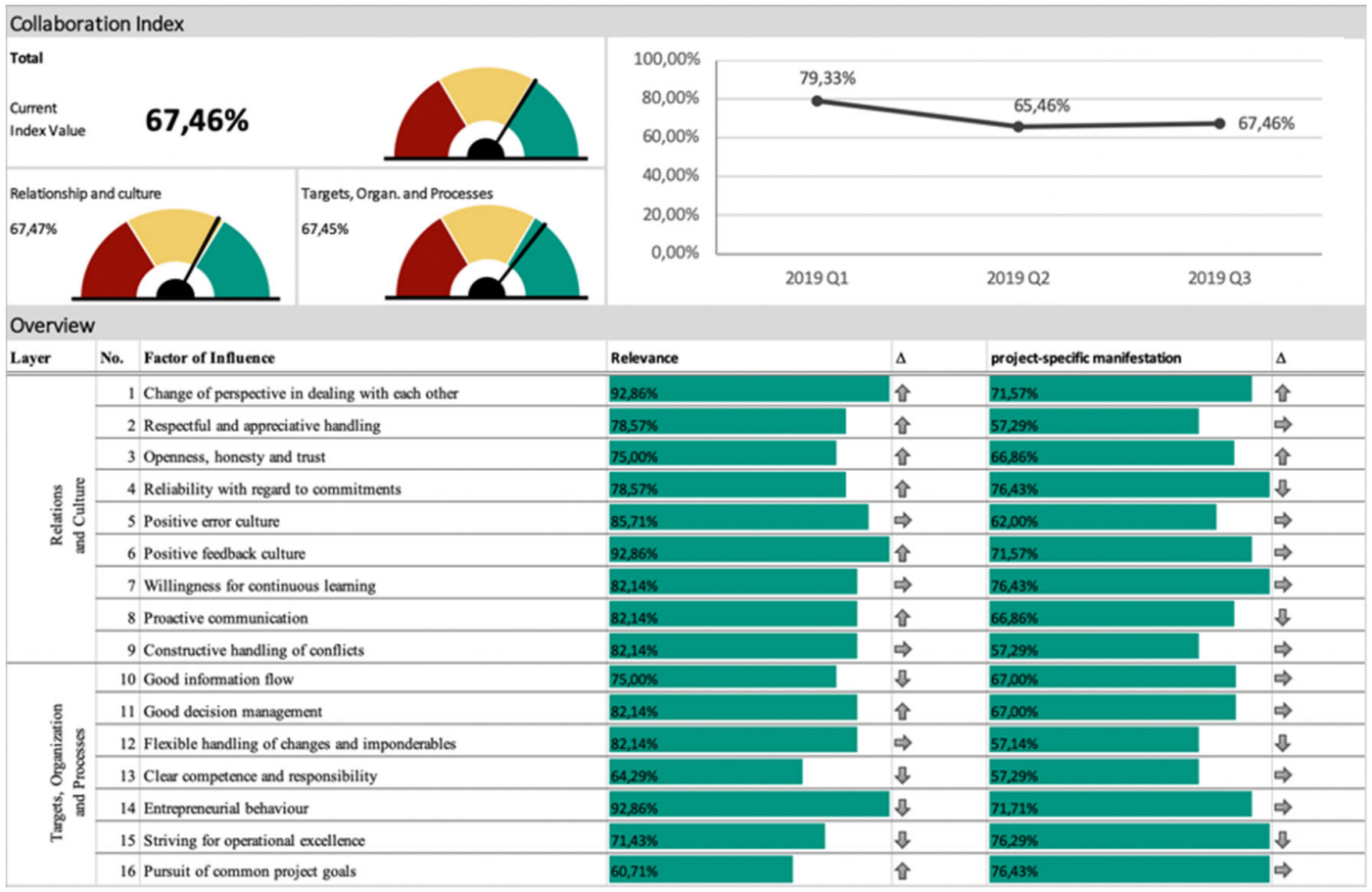

Figure 5: Exemplary extract from the evaluation of the collaboration barometer 
While the upper half of the collaboration barometer provides a direct overview of the current status and progress of the collaboration, the lower part can be used for detailed analysis. In the present example (figure 5) it is shown that, for example, a positive error culture (No. 5) is very important, but that the implementation in the project has weaknesses.

For most applications, the survey was conducted quarterly. The project teams used it especially as a basis for discussion within the project meetings to identify and discuss suggestions for improvement. A major advantage was the specific evaluations that were made for each project group. In this way, numerous communication deficits were uncovered and countermeasures were developed.

\section{CONCLUSIONS}

In this paper, the results of a research project were presented, in the context of which a method was developed that allows to record and evaluate the cooperation in a project. The term "collaboration" was first explained, before in the next step possibilities for measuring collaboration were shown in order to derive a measurement method based on this. As a result, a questionnaire was created which can be used in the context of a given process in the company of a third party, such as an university. The results of the first application show that there is a high level of acceptance among the participants and that they are convinced of the advantages of the measurement instrument.

In the future, the results of the collaboration barometer could be used in a larger number of projects to make more substantiated statements about the influence of collaboration on the project outcome. The current data basis is not yet sufficient for this.

\section{REFERENCES}

Abdirad, H. and Pishdad-Bozorgi, P. (2014). "Developing a Framework of Metrics to Assess Collaboration in Integrated Project Delivery". 50th ASC Annual International Conference Proceedings. Blacksburg, Virginia, USA.

Anvuur, A. M. (2008). Cooperation in Construction Projects: Concept, Antecedents and Strategies. PhD Thesis. University of Hong Kong. New York. Taylor \& Francis.

Axelrod, R. M. (2000). "Die Evolution der Kooperation“. München, Oldenbourg.

Bierhoff, H. W. (1998). "Sozialpsychologische Aspekte der Kooperation". Spieß, E., (editor). "Formen der Kooperation. Bedingungen und Perspektiven." Göttingen, Verlag für Angewandte Psychologie, p. 21-35.

El Asmar, M. (2012). "Modeling and Benchmarking Performance for the Integrated Project Delivery (IPD) System". PhD Thesis. University of Wisconsin. Madison, US.

Emmitt, S. and Gorse, C. A. (2007). "Communication in Construction Teams". London. Routledge. https://doi.org/10.4324/9780203018798.

Fischer, M., Ashcraft, H. W., Reed, D., Khanzode, A (2017). "Integrating Project Delivery". John Wiley \& Sons. Somerset.

Girmscheid, G. (2010). Strategisches Bauunternehmensmanagement. Prozessorientiertes integriertes Management für Unternehmen in der Bauwirtschaft. Heidelberg, Springer.

Langemeyer, I. (2015). "Das Wissen der Achtsamkeit. Kooperative Kompetenz in komplexen Arbeitsprozessen“. Münster, Waxmann.

Merkle, A. (2017). "Baukonflikte verstehen und umgehen. Neue Wege der Kooperation". Fraunhofer IRB. Stuttgart, Germany. 
Nerdinger, F. W. (1998). "Extra-Rollenverhalten und Kooperation in Organisationen". Spieß, E., (editor). „Formen der Kooperation. Bedingungen und Perspektiven“. Göttingen, Verlag für Angewandte Psychologie, p. 265-278.

Noack, J. and Schienmann, B. (1999). "Objektorientierte Vorgehensmodelle im Vergleich". Informatik-Spektrum 22. p. 166-180.

Österreichische Bautechnik Vereinigung (2018). "Kooperative Projektabwicklung". https:/www.bautechnik.pro/DE/Shop/artikeldetail?IDArtikel=b66f68bd-cdf2-46f086b3- 3ebffa1d2eae (09.04.2019).

Phua, F.T.T. (2004). "The antecedents of co-operative behaviour among project team members: an alternative perspective on an old issue". Construction Management and Economics, 22(10), 1033-1045.

Pocock, J., Hyun, C., Liu, L. and Kim, M. (1996). "Relationship between Project Interaction and Performance Indicators". Journal of Construction Engineering and Management, 122(2), 165-176. doi: 10.1061/(ASCE)0733-9364(1996)122:2(165)

Schöttle, A., Haghsheno, S. and Gehbauer, F. (2014). "Defining Cooperation and Collaboration in the Context of Lean Construction". Proceedings IGLC-22. 12691280. Oslo, Norway.

Spang, K. (2006). "Innovative Projektabwicklung bei Bauprojekten - Plädoyer für einen Paradigmenwechsel“. Der Bauingenieur, 81, p.117-125.

Spieß, E. (1998a). "Das Konzept der Empathie“. Spieß, E., (editor). „Formen der Kooperation. Bedingungen und Perspektiven". Göttingen, Verlag für Angewandte Psychologie, p. 53-61.

Spieß, E., (editor). (1998b). "Formen der Kooperation. Bedingungen und Perspektiven". Göttingen, Verlag für Angewandte Psychologie.

Tries, J. and Reinhardt, R. (2008). "Konflikt- und Verhandlungsmanagement. Konflikte konstruktiv nutzen“. Berlin, Heidelberg, Springer.

Walker, D. H. T., Lloyd-Walker, B. (2019). "Characteristics of IPD". Walker, D., Rowlinson, S. (editor). „Routledge Handbook of Integrated Project Delivery“. New York, Routledge, p. 20-40. 